\title{
Using IEC 61508 to Guide the Investigation of Computer-Related Incidents and Accidents
}

\author{
Chris Johnson, \\ Dept. of Computing Science, University of Glasgow, Glasgow, G12 9QQ. \\ Tel.: +44 141330 6053, Fax: +44 1413304913 \\ johnson@dcs.gla.ac.uk
}

\begin{abstract}
Relatively few investigation techniques have been specifically developed to identify the causal factors that contribute to mishaps involving safety-critical computer systems. This is a significant omission because a number of factors distinguish this class of incidents from other mishaps. For example, the Rand report into NTSB investigation methods observed that the introduction of software control systems has greatly increased the integration and complexity of many applications. This has had 'knock-on' effects in terms of the complexity of any incident investigation. The following pages, therefore, present two complementary investigation techniques that are intended to support the analysis of Electrical, Electronic or Programmable, Electronic Systems (E/E/PES)-related mishaps. One is intended to provide a low-cost and lightweight approach that is appropriate for low consequence events. It is based around a flowchart that prompts investigators to identify potential causal factors through a series of questions about the events leading to a failure and the context in which they occurred. The second approach is more complex. It involves additional documentation and analysis. It is, therefore, more appropriate for incidents that have greater potential consequences or a higher likelihood of recurrence. This approach uses Events and Causal Factors (ECF) modelling together with particular forms of causal reasoning developed by the US Department of Energy (1992). Both approaches provide means of mapping causal factors back to the lifecycle phases and common requirements described in the IEC 61508 standard. This provides an important bridge from the products of mishap analysis to the design and operation of future systems. The UK Health and Safety Executive sponsored this work as part of an initiative to develop analysis techniques for E/E/PES related incidents. The events leading to an explosion and fires in a fractional distillation unit are used to illustrate the application of our techniques.
\end{abstract}

\section{Introduction}

Very few accident analysis techniques support the investigation of adverse events involving programmable systems. There are some notable exceptions, including Leveson's (2002) STAMP and the Why-Because Analysis proposed by Ladkin and Loer (1998). Unfortunately, these techniques provide limited support for the generation of recommendations. They say little about possible intervention in the software or hardware development processes. In contrast, this paper presents two causal analysis techniques that are well integrated with development techniques for E/E/PES-related systems. In particular, we focus on methods for using the findings of incident investigations to inform the application of the IEC 61508 standard. This approach is justified by the current commercial acceptance of 61508 , although both of our approaches can be integrated with other standards.

\section{The Case Study Incident}

The following pages describe an incident involving a fluidised catalytic cracking unit, part of a UK refinery complex, further details can be found in the official report into this incident (HSE, 1997). The plant receives crude oil, which is then separated by fractional distillation into intermediate products, including light and heavy diesel, naptha, kerosese and other heavier components. These heavier elements are eventually fed into the fluidised catalytic cracking unit. This is a continuous process to convert 'long' 
chain hydrocarbons into smaller hydrocarbon products used in fuels. The immediate events leading to the incident started when lightning started a fire in part of the crude distillation unit within the plant. This led to a number of knock-on effects, including power disruption, which affected elements in the fluidised catalytic cracking unit. Initially, hydrocarbon flow was lost to the deethaniser, illustrated in Figure 1. This caused the liquid in the vessel to empty into the next stage debutanizer. The control system was programmed to prevent total liquid loss in these stages and so valve A was closed. This starved the debutanizer of feed. The programmable system again intervened to close valve B. The liquid trapped in the debutanizer was still being heated even though both valves now isolated it. Pressure rose and the vessel vented to a flare. Shortly afterwards, the liquid level in the deethaniser was restored, the control system opened valve A and the debutanizer received further flow. Valve B should have opened at this time to allow fluid from the pressurised debutanizer into the naptha splitter. Operators in the control room received misleading signals that valve $B$ had been successfully reopened by their control system even though this had not occurred. As a result the debutanizer filled with liquid while the naptha splitter was emptied.

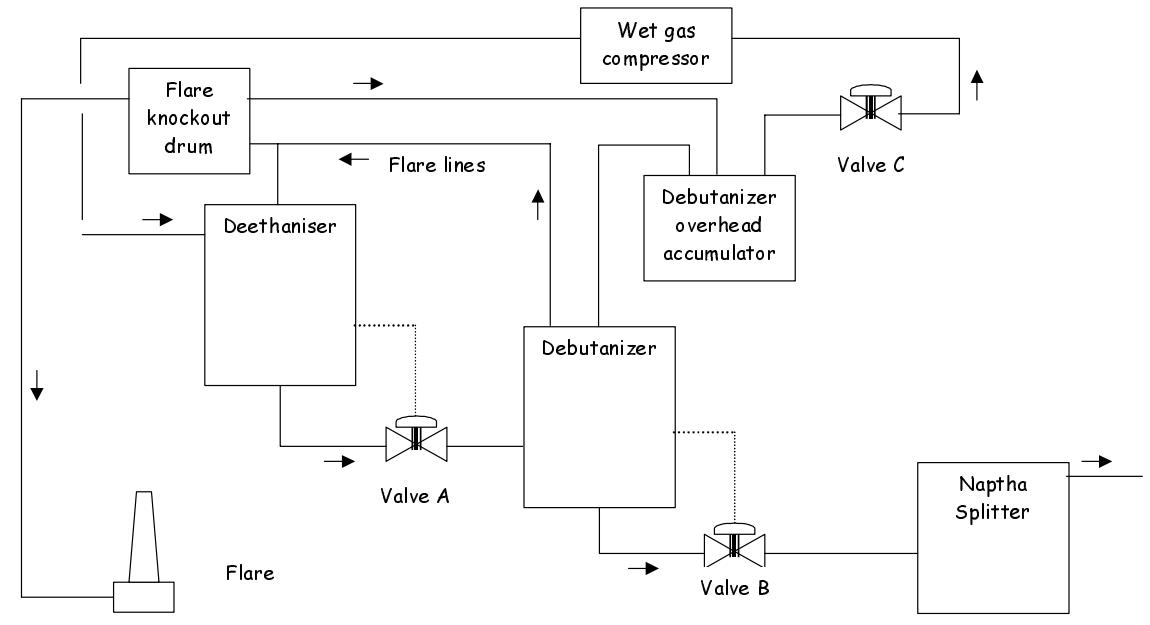

Fig. 1. High-level Overview of Components in the Fluidised Catalytic Cracking Unit

The control room displays separated crucial information that was necessary to diagnose the source of the rising pressure in the debutanizer. Rather than checking the status of valve $\mathrm{B}$, the operators took action to open valve $\mathrm{C}$. This allowed liquid in the full overhead accumulator to flow back into a recovery section of the plant but was insufficient to prevent the debutanizer from becoming logged with fluid entering from the deethanizer. Again, the debutanizer vented to the flare line. Opening valve $\mathrm{C}$ created a flow of fluid into previous 'dry' stages of the process that eventually caused a compressor trip. Large volumes of gas now had nowhere to go within the process and had to be vented to the flare stack to be burned off. At this stage, the volume of materials in the flare knockout drum was further increased by attempts to use fire hoses to drain the flooding from the dry stage directly into the flare line. However, this enabled the wet gas compressor to be restarted. This should have made matters better by increasing the flow of materials through the unit but had the unwanted effect of causing a further increase of pressure in the debutanizer. The operators responded again by opening valve $\mathrm{C}$ causing a further trip of the compressor. More materials were vented to an already full flare drum. Liquid was forced into a corroded discharge pipe, which broke at an elbow bend causing 20 tonnes of highly flammable hydrocarbon to be discharged. The resulting vapour cloud ignited causing damage estimated to be in excess of $£ 50$ million.

This case study has been chosen to illustrate the remainder of the paper because it is typical of the way in which incidents stem from the interaction between E/E/PES-related failures, operator 'error', hardware faults and management issues. Figure 2 illustrates both the stages in our proposed analysis techniques and also the structure for the remainder of the paper. A section on information elicitation is followed by detailed discussions of our two proposed techniques. Later sections describe how recommendations can be 
derived from the results of a causal analysis. The closing sections of this paper identify a number of conclusions and areas for further work.

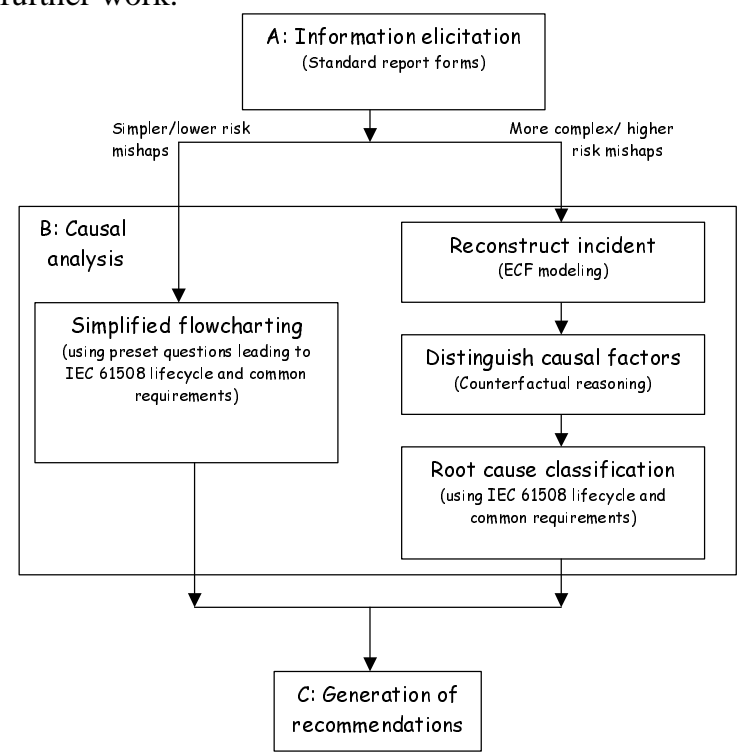

Fig. 2. Structure of the paper and an overview of the two candidate investigation schemes

\section{Elicitation}

Incident reporting forms need to be specifically tailored to elicit information about E/E/PES-related failures. For example, end-users who initially observe a failure may have little reason to suspect the involvement of programmable systems. In such circumstances, reporting forms should prompt operators to consider the involvement of such systems and take appropriate actions. These can include the preservation of automated logs and data sources. Similarly, reporting forms can be revised to request details about both hardware and software version numbers. Such distinctions are not routinely made in existing forms but can be crucial when reporting adverse events back to manufacturers and regulators. The nature of the information obtained will largely be determined by their knowledge of the systems involved. For instance, someone involved in the development or integration of an E/E/PES will be able to provide additional detail and insight beyond that which might normally be expected of a system operator. Conversely, someone involved in the operation of the application can provide information about the previous operating history that might not be available to system developers. Different forms must be developed to elicit the different information available to these different groups of people. Brevity prevents a detailed discussion of form design for the elicitation of information about computer-related mishaps. This topic is discussed and sample forms are provided in Emett et al (2002). Additional requirements for the processing of system logs and other forms of automated records that must be safeguarded in the aftermath of an incident are discussed in Johnson, Le Galo and Blaize (2000).

\section{Root Causes of E/E/PES Related Incidents Under IEC 61508}

Most computer-related incidents stem from problems in the development lifecycle. Latent causes occur in risk assessment, design, implementation, testing, maintenance etc. Other problems, such as poor project management; affect many stages of development. It is for this reason that both of the causal analysis techniques in this paper exploit the lifecycle and process requirements embedded within the IEC 61508 standard. This standard is one of several that could have been used (Johnson, 2003). The decision to 
adopt this standard is justified by its relatively widespread adoption for E/E/PES development within the process industries. The UK Health and Safety Executive have identified this application area as a focus for our work. Table 1 provides a high-level classification of the potential problems that affect phases of the IEC 61508 lifecycle or the common requirements that hold across several phases. These issues are enumerated in the middle column. The right column provides a reference to areas of the standard that provide additional detail about each requirement. The rows in this table will be used in the remainder of this report to provide a taxonomy or checklist of causal factors. As our analysis progresses we will attempt to identify which of these potential failures contributed to the particular causes of our case study.

\section{Flow Charting Scheme}

Figures 3 and 4 provide an overview of our flow-charting technique ${ }^{1}$. Analysis begins by asking a series of high-level questions about the nature of the E/E/PES-related incident. Investigators must determine whether or not the system correctly intervened to prevent a hazard, as might be the case in a near miss incident. If the answer is yes, then the analysis progresses by moving horizontally along the arrows to identify the nature of the failure. If the system intervened to address problems created by maintenance activities then the investigator would follow the arrow in Figure 3 down to the associated table entry. By reading each cell in the column of the table indicated by the arrow, investigators can identify potential causes in the simplified stages of the IEC 61508 lifecycle. Latent failures that might have been the source of computer-related incident could also be considered by examining the items listed under all six of the common requirements in the third row from the bottom. Investigators continue along the top horizontal line repeating the classification against the cells in the table in the same manner described for maintenance related incidents. Analysis progresses by following the top-level questions down the flow chart. For some incidents, there will be failures identified by analyzing several of these different questions. A system may operate correctly to prevent a hazard although in the process there may also be further subsystem failures or operator interventions that initially fail to rectify the situation. In this case, analysts would focus on the top line in Figure 3 and the further line of analysis continued on Figure 4.

It is difficult to justify this exhaustive form of analysis for relatively minor incidents. In such cases, investigators may choose to stop once they have identified an initial selection of potential causes from the IEC 61508 flowcharts. In this case, it is important that Safety Managers consider the order of questions in Figures 3 and 4. For instance, the current format asks whether maintenance issues potentially caused an incident before it elicits information about operator failures. This ordering can bias partial analyses towards the initial causal factors. It is for this reason that we recommend a more sustained and exhaustive analysis of the flow charts. If this is not possible then safety managers should monitor the products of any causal analysis to identify the effects of any potential ordering bias.

The flowcharts illustrated in Figures 3 and 4 have been validated against a series of case study incidents. These were identified by the Health and Safety Executive as in some way 'typical' of the E/E/PES related failures that occur in the process industries. Each of the incidents that we have examined has helped to drive further refinements to the flowchart. This process is continuing as we have now begun a series of usability studies and validation exercises involving safety managers from across the process industries, including nuclear power generation and petrochemical production. These validation exercises also include participation from companies who supply and integrate E/E/PES applications. This is important because they are often called upon to identify the causes of mishaps that are reported by end-users. It is expected that further revisions will be made to the flowcharts as a result of this consultation exercise. However, Figures 3 and 4 do provide an indication of the general approach that we have adopted to support the analysis of less complex incidents and accidents.

\footnotetext{
${ }^{1}$ Initial ideas for this technique were provided by Bill Black and are documented in Emmet et al (2002).
} 


\begin{tabular}{|c|c|c|}
\hline $\begin{array}{c}\text { IEC } 61508 \\
\text { Lifecycle phase }\end{array}$ & Detailed taxonomy & IEC 61508 ref \\
\hline Concept & \multirow{3}{*}{$\begin{array}{l}\text { 1. Hazard identification } \\
\text { 2. Consequence and likelihood estimation }\end{array}$} & \multirow[t]{3}{*}{$7.2,7.3,7.4$} \\
\hline Overall Scope & & \\
\hline $\begin{array}{l}\text { Hazard \& } \\
\text { Risk Assessment }\end{array}$ & & \\
\hline Overall Safety & \multirow{5}{*}{$\begin{array}{l}\text { 1.specification } \\
\text { 2. selection of equipment } \\
\text { 3. design and development } \\
\text { 4. installation design } \\
\text { 5. maintenance facilities } \\
\text { 6. operations facilities }\end{array}$} & \multirow{5}{*}{$\begin{array}{c}7.2(2) \\
7.4 .2 .2(2) \\
7.4(2) \\
7.4 .4 / 5(2) \\
7.4 .4 .3(2), \\
7.4 .5 .2 / 3(2) \\
7.4 .5 .1 / 3\end{array}$} \\
\hline Requirements & & \\
\hline Allocation & & \\
\hline $\begin{array}{l}\text { Planning of I \& } \mathrm{C} \text {, } \\
\mathrm{V}, \text { and } \mathrm{O} \& \mathrm{M}\end{array}$ & & \\
\hline Realization & & \\
\hline $\begin{array}{l}\text { Installation and } \\
\text { commissioning }\end{array}$ & $\begin{array}{ll}\text { 1. } & \text { installation } \\
\text { 2. } & \text { commissioning }\end{array}$ & $\begin{array}{ll}7.5 & (2), \\
7.13 .2 .1 / 2, & \\
7.13 .2 .3 / 4 & \end{array}$ \\
\hline Validation & $\begin{array}{ll}\text { 1. } & \text { function testing } \\
\text { 2. } & \text { discrepancies analysis } \\
\text { 3. } & \text { validation techniques }\end{array}$ & $\begin{array}{l}7.7 .2 .1 / 2 / 3(2) \\
7.7 .2 .5(2) \\
7.7 .2 .7(2)\end{array}$ \\
\hline $\begin{array}{l}\text { Operation and } \\
\text { maintenance }\end{array}$ & $\begin{array}{l}\text { 1. maintenance procedures not applied } \\
\text { 2. maintenance procedures need improvement } \\
\text { 3. operation procedures not applied } \\
\text { 4. operations procedures need improvement } \\
\text { 5. permit/hand over procedures } \\
\text { 6. test interval not sufficient } \\
\text { 7. maintenance procedures not impact assessed } \\
\text { 8. operation procedures not assessed } \\
\text { 9. LTA procedures to monitor system performance } \\
\text { 10. LTA procedures applied to initiate modification in the event of systematic } \\
\text { 11. tools incorrectly selected or not applied correctly }\end{array}$ & $\begin{array}{l}\text { 7.7.2.1 } \\
7.6 .2 .2 .1 / 2 / 3(2) \\
7.6 .2 .1 \\
7.6 .2 .2 \\
7.6 .2 .1 \\
7.6 .2 .1 \\
7.6 .2 .4(2) \\
7.6 .2 .4(2) \\
7.6 .2 .1(2) \\
7.8 .2 .2 \\
7.16 .2 .2 \\
7.6 .2 .1(2)\end{array}$ \\
\hline Modification & $\begin{array}{l}\text { 1. impact analysis incorrect } \\
\text { 2. LTA manufacturers information } \\
\text { 3. full lifecycle not implemented } \\
\text { 4. } \text { LTA verification and validation }\end{array}$ & $\begin{array}{l}7.8 .2 .1(2) \\
7.8 .2 .2(2) \\
7.8 .2 .3(2) \\
7.8 .2 .4(2)\end{array}$ \\
\hline \multicolumn{3}{|c|}{ IEC 61508 common requirements } \\
\hline Competency & $\begin{array}{ll}\text { 1. } & \text { LTA operations competency } \\
\text { 2. } & \text { LTA maintenance competency } \\
\text { 3. } & \text { LTA modification competency }\end{array}$ & $\begin{array}{l}6.2 .1 \mathrm{~h} \\
6.2 .1 \mathrm{~h} \\
6.2 .1 \mathrm{~h}\end{array}$ \\
\hline Lifecycle & $\begin{array}{l}\text { 1. LTA definition of operations accountabilities } \\
\text { 2. LTA definition of maintenance accountabilities } \\
\text { 3. } \quad \text { LTA definition of modification accountabilities }\end{array}$ & $\begin{array}{l}7.1 .4 \\
7.1 .4 \\
7.1 .4\end{array}$ \\
\hline Verification & 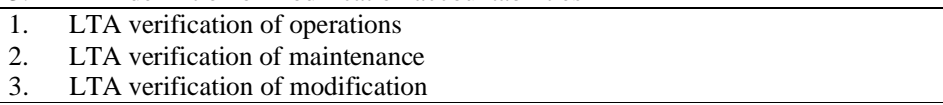 & $\begin{array}{l}7.18 .2,7.9(2) \\
7.18 .2,7.9(2) \\
7.18 .2,7.9(2)\end{array}$ \\
\hline $\begin{array}{l}\text { Safety } \\
\text { management }\end{array}$ & $\begin{array}{l}\text { 1. LTA safety culture } \\
\text { 2. LTA safety audits } \\
\text { 3. } \quad \text { LTA management of suppliers } \\
\end{array}$ & $\begin{array}{l}6.2 .1 \\
6.2 .1 \\
6.2 .5\end{array}$ \\
\hline Documentation & $\begin{array}{l}\text { 1. documentation unclear or ambiguous } \\
\text { 2. documentation incomplete } \\
\text { 3. documentation not up to date }\end{array}$ & $\begin{array}{l}5.2 .6 \\
5.2 .3 \\
5.2 .11\end{array}$ \\
\hline $\begin{array}{l}\text { Functional safety } \\
\text { assessment }\end{array}$ & $\begin{array}{ll}\text { 1. } & \text { LTA O \& M assessment } \\
\text { 2. } & \text { modification assessment LTA } \\
\text { 3. } & \text { assessment incomplete } \\
\text { 4. } & \text { insufficient skills or independence in assessment team }\end{array}$ & $\begin{array}{l}8.2 \\
8.2 \\
8.2 .3 \\
8.2 .11 / 12 / 13 / 14\end{array}$ \\
\hline
\end{tabular}

Key: LTA is Less Than Adequate, IEC 61508 references are to Part 1 except as indicated by parentheses e.g. (2)

Table 1. Taxonomy for Analyzing Computer Related Failures Under IEC 61508 (Emmet et al 2003). 


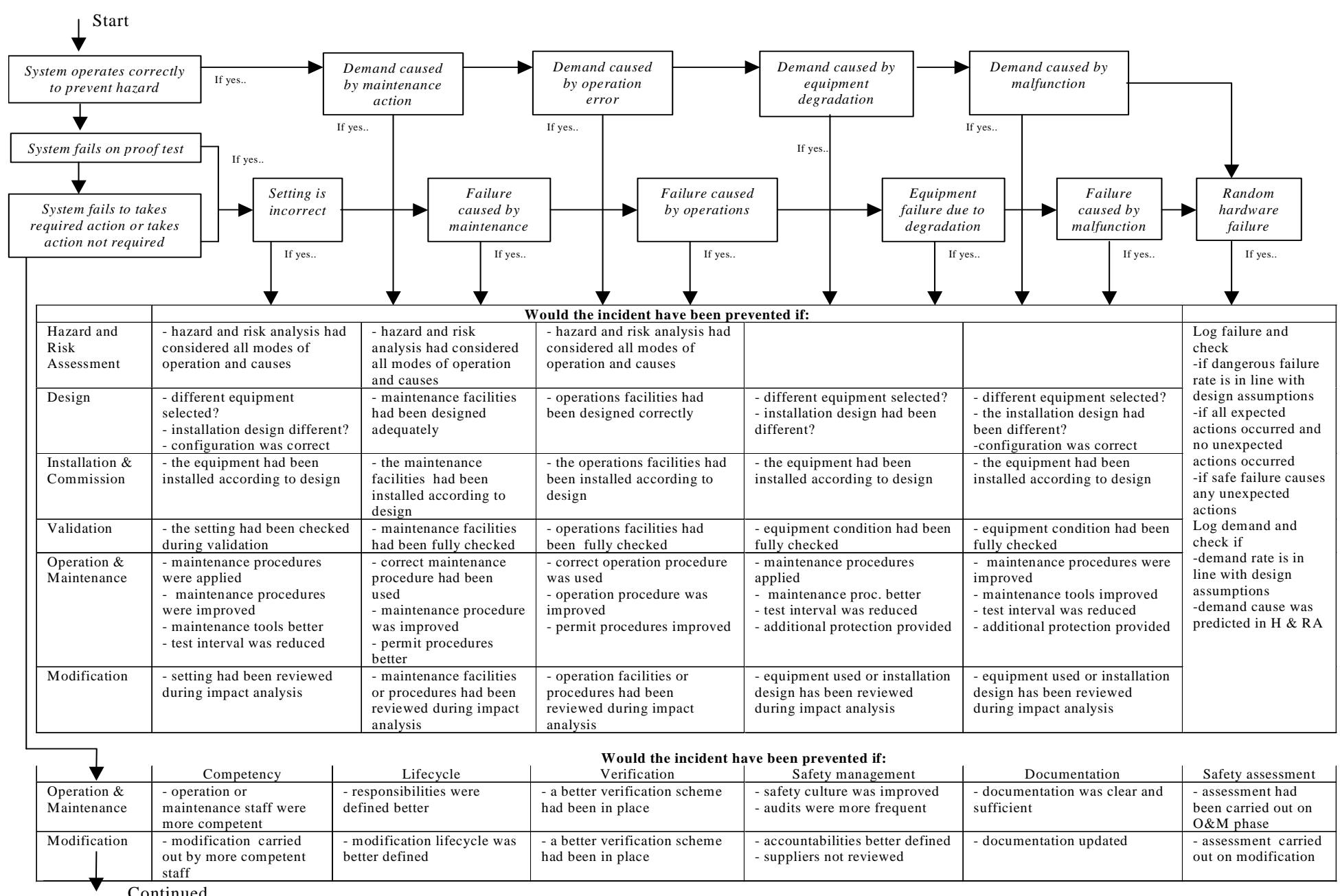

Fig. 3. High-Level Flow Chart to Support Causal Analysis of E/E/PES Related Incidents Using IEC 61508 Taxonomy [Cont. in next figure] (Emmet et al, 2003) 
Continued from previous figure

\begin{tabular}{|c|c|c|c|c|c|c|c|c|c|c|c|}
\hline \multirow[t]{2}{*}{$\begin{array}{c}\text { Incorrect } \\
\text { action taken } \\
\text { by system or } \\
\text { operator }\end{array}$} & \multirow[t]{2}{*}{$\overrightarrow{\text { If yes.. }}$} & $\begin{array}{l}\text { No action by } \\
\text { operator allows } \\
\text { demand on } \\
\text { system }\end{array}$ & \multicolumn{2}{|c|}{$\begin{array}{l}\text { System actions } \\
\text { insufficient to } \\
\text { terminate } \\
\text { hazard }\end{array}$} & \multicolumn{2}{|l|}{$\begin{array}{c}\text { System takes } \\
\text { unnecessary } \\
\text { actions }\end{array}$} & \multicolumn{2}{|c|}{$\begin{array}{l}\text { No mitigation } \\
\text { takes place }\end{array}$} & \multicolumn{2}{|c|}{$\begin{array}{l}\text { Operator fails to } \\
\text { mitigate hazard }\end{array}$} & \\
\hline & & If yes.. & \multicolumn{2}{|l|}{ If yes.. } & \multicolumn{2}{|l|}{ If yes.. } & \multicolumn{2}{|c|}{ If yes.. } & \multicolumn{2}{|c|}{ If yes.. } & \\
\hline & \multicolumn{9}{|c|}{ Would the incident have been prevented if: } & & \\
\hline $\begin{array}{l}\text { Hazard and } \\
\text { Risk } \\
\text { Assessment }\end{array}$ & \multicolumn{2}{|c|}{$\begin{array}{l}\text { - hazard \& risk } \\
\text { analysis had } \\
\text { considered all modes } \\
\text { of operation \& causes }\end{array}$} & $\begin{array}{l}\text { - hazard \& risk analysis } \\
\text { had considered all modes } \\
\text { of operation \& causes }\end{array}$ & \multicolumn{2}{|c|}{$\begin{array}{l}\text { - hazard \& risk analysis had } \\
\text { considered all modes of } \\
\text { operation \& causes }\end{array}$} & \multicolumn{2}{|c|}{$\begin{array}{l}\text { - hazard \& risk analysis } \\
\text { had considered all modes } \\
\text { of operation \& causes }\end{array}$} & \multicolumn{2}{|c|}{$\begin{array}{l}\text { - hazard \& risk analysis } \\
\text { had considered all modes } \\
\text { of operation \& causes }\end{array}$} & \multirow{6}{*}{\multicolumn{2}{|c|}{$\begin{array}{l}\text { Log failure and check } \\
\text {-if dangerous failure rate } \\
\text { is in line with design } \\
\text { assumptions } \\
\text {-if all expected actions } \\
\text { occurred and no } \\
\text { unexpected actions } \\
\text { occurred } \\
\text {-if safe failure causes any } \\
\text { unexpected actions } \\
\text { Log demand and check if } \\
\text { - demand rate is in line } \\
\text { with design assumptions } \\
\text { - demand cause was } \\
\text { predicted in H \& RA }\end{array}$}} \\
\hline Design & \multicolumn{2}{|c|}{\begin{tabular}{|c|} 
- operator facilities \\
wer designed better \\
- installation design \\
had been different?
\end{tabular}} & $\begin{array}{l}\text { - additional actions were } \\
\text { specified } \\
\text { - actions were faster } \\
\text { - final actuation device } \\
\text { were improved }\end{array}$ & \multicolumn{2}{|c|}{$\begin{array}{l}\text { - design requirements were } \\
\text { better documented }\end{array}$} & \multicolumn{2}{|c|}{$\begin{array}{l}\text { - mitigation system had } \\
\text { been specified } \\
\text { - mitigation system had } \\
\text { been better designed }\end{array}$} & \multicolumn{2}{|c|}{$\begin{array}{l}\text { - operator facilities had } \\
\text { been designed better } \\
\text { - the installation design } \\
\text { had been different? }\end{array}$} & & \\
\hline $\begin{array}{l}\text { Installation \& } \\
\text { Commissioning }\end{array}$ & \multicolumn{2}{|c|}{$\begin{array}{l}\text { - equipment had been } \\
\text { installed according to } \\
\text { design }\end{array}$} & $\begin{array}{l}\text { - the equipment had been } \\
\text { installed according to } \\
\text { design }\end{array}$ & \multicolumn{2}{|c|}{$\begin{array}{l}\text { - the equipment had been } \\
\text { installed according to design }\end{array}$} & \multicolumn{2}{|c|}{$\begin{array}{l}\text { - mitigation system had } \\
\text { been installed according } \\
\text { to design }\end{array}$} & \multicolumn{2}{|c|}{$\begin{array}{l}\text { - equipment had been } \\
\text { installed according to } \\
\text { design }\end{array}$} & & \\
\hline Validation & \multicolumn{2}{|c|}{\begin{tabular}{|l|} 
- operator facilities \\
had been checked \\
during validation
\end{tabular}} & $\begin{array}{l}\text { - operation facilities had } \\
\text { been checked during } \\
\text { validation }\end{array}$ & \multicolumn{2}{|c|}{$\begin{array}{l}\text { - operations facilities had } \\
\text { been fully checked }\end{array}$} & \multicolumn{2}{|c|}{$\begin{array}{l}\text { - mitigation system had } \\
\text { been fully checked }\end{array}$} & \multicolumn{2}{|c|}{$\begin{array}{l}\text { - operator facilities had } \\
\text { been fully checked }\end{array}$} & & \\
\hline $\begin{array}{l}\text { Operation \& } \\
\text { Maintenance }\end{array}$ & \multicolumn{2}{|c|}{$\begin{array}{l}\text { - operation procedures } \\
\text { were applied } \\
\text { - operation } \\
\text { procedures were } \\
\text { improved }\end{array}$} & $\begin{array}{l}\text { - correct maintenance } \\
\text { procedure had been used } \\
\text { - maintenance procedure } \\
\text { was improved } \\
\text { - proof testing was more } \\
\text { frequent }\end{array}$ & \multicolumn{2}{|c|}{$\begin{array}{l}\text { - correct operation } \\
\text { procedure was used } \\
\text { - operation procedure was } \\
\text { improved } \\
\text { - permit procedures were } \\
\text { improved }\end{array}$} & & $\begin{array}{l}\text { gation procedures } \\
\text { applied } \\
\text { gation procedures } \\
\text { mproved } \\
\text { gation system was } \\
\text { checked more } \\
\text { ntly }\end{array}$ & $\begin{array}{l}\text { - operat } \\
\text { had bee } \\
\text { - operat } \\
\text { procedu } \\
\text { improve }\end{array}$ & $\begin{array}{l}\text { procedures } \\
\text { pplied } \\
\text { facilities or } \\
\text { were }\end{array}$ & & \\
\hline Modification & $\begin{array}{l}\text {-oper } \\
\text { had b } \\
\text { durin }\end{array}$ & $\begin{array}{l}\text { ation facilities } \\
\text { een reviewed } \\
\text { impact analysis }\end{array}$ & $\begin{array}{l}\text { necessary system } \\
\text { actions had been } \\
\text { reviewed during impact } \\
\text { analysis }\end{array}$ & $\begin{array}{l}\text { - neces } \\
\text { had bec } \\
\text { impact }\end{array}$ & $\begin{array}{l}\text { y system actions } \\
\text { reviewed during } \\
\text { alysis }\end{array}$ & & $\begin{array}{l}\text { for mitigation had } \\
\text { eviewed during } \\
t \text { analysis }\end{array}$ & $\begin{array}{l}\text { - need } \\
\text { been re } \\
\text { impact }\end{array}$ & $\begin{array}{l}\text { nitigation had } \\
\text { ved during } \\
\text { lysis }\end{array}$ & & \\
\hline & & & & & If the & & & & & & \\
\hline$\nabla$ & & Competency & Lifecycle & & Verification & & Safety manager & & Documentati & & Safety assessment \\
\hline $\begin{array}{l}\text { Operation \& } \\
\text { Maintenance }\end{array}$ & $\begin{array}{l}\text { - oper } \\
\text { staff }\end{array}$ & $\begin{array}{l}\text { ation or maintenance } \\
\text { vere more competent }\end{array}$ & $\begin{array}{l}\text { - responsibilities were } \\
\text { better }\end{array}$ & fined & $\begin{array}{l}\text { - a better verification } \\
\text { scheme had been in } \mathrm{p}\end{array}$ & & $\begin{array}{l}\text { - safety culture was } i \\
\text { - audits were more } \mathrm{fr}\end{array}$ & $\begin{array}{l}\text { proved } \\
\text { puent }\end{array}$ & $\begin{array}{l}\text { - documentatior } \\
\text { clear and suffic }\end{array}$ & & $\begin{array}{l}\text { - assessment had } \\
\text { been carried out on } \\
\text { O\&M phase }\end{array}$ \\
\hline Modification & $\begin{array}{l}\text { - mod } \\
\text { carrie } \\
\text { comp }\end{array}$ & $\begin{array}{l}\text { ification had been } \\
\text { d out by more } \\
\text { etent staff }\end{array}$ & $\begin{array}{l}\text { - modification lifecycl } \\
\text { better defined }\end{array}$ & & $\begin{array}{l}\text { - a better verification } \\
\text { scheme had been in } \mathrm{p}\end{array}$ & & $\begin{array}{l}\text { - accountabilities we } \\
\text { defined } \\
\text { - suppliers not revier }\end{array}$ & $\begin{array}{l}\text { better } \\
\text { d }\end{array}$ & $\begin{array}{l}\text { - documentatior } \\
\text { been updated }\end{array}$ & & $\begin{array}{l}- \text { assessment ad } \\
\text { been carried out on } \\
\text { modification }\end{array}$ \\
\hline
\end{tabular}

Fig. 4. High-Level Flow Chart to Support Causal Analysis of E/E/PES Related Incidents Using IEC 61508 Taxonomy (Emmet et al, 2003) 
Most incidents involve multiple causes. Our case study, amongst other things, stemmed from the operators decision to open valve $\mathrm{C}$ as a means of decreasing pressure in the debutanizer whilst failing to notice that the E/E/PES had failed to open valve B. Their decision was informed by erroneous information from their control system, which indicated that valve B was open and from a sensor malfunction that indicated the flow and level in the debutanizer had not reached their maximum values. These problems were compounded by poor interface design. Fractal distillation takes one primary source material and produces five product streams. Critical information about the volume of production on each of these streams was distributed across several displays. The analysis might identify several requirements or lifecycle activities that might have prevented this incident from occurring in the manner described. It is important to document the outcome of this flowchart analysis. This is done using the form illustrated in Table 2. Immediate events that are identified in incident reporting forms are related back to failures in the lifecycle stages and common requirements of IEC 61508. This allocation process is guided by the questions in Figures 3 and 4 . The allocation is also supported by a justification that is intended to document any intermediate reasoning to other investigators and co-workers.

\begin{tabular}{|c|c|c|c|}
\hline $\begin{array}{l}\text { Causal } \\
\text { Event } \\
\end{array}$ & $\begin{array}{l}\text { IEC } 61508 \\
\text { Classification }\end{array}$ & Route through flow chart & Rationale \\
\hline $\begin{array}{l}\text { Decision to } \\
\text { open } \\
\text { valve } C \text {. }\end{array}$ & Validation & $\begin{array}{l}\text { Incorrect action taken by } \\
\text { system or operator-> } \\
\text { Operator fails to mitigate } \\
\text { hazard -> } \\
\text { Accident would have been } \\
\text { avoided if operator facilities } \\
\text { had been fully checked. }\end{array}$ & $\begin{array}{l}\text { The operators intervened in the automated } \\
\text { control system to open valve } \mathrm{C} \text { this twice } \\
\text { led the compressor to trip and forced } \\
\text { excess fluid into the flare system. The } \\
\text { poorly designed displays prevented them } \\
\text { from diagnosing the source of the } \\
\text { increased pressure in the debutanizer and } \\
\text { the potential hazard from their actions in } \\
\text { opening C. Improved display design might } \\
\text { have occurred if they had been validated } \\
\text { against a wider range of operational } \\
\text { scenarios. }\end{array}$ \\
\hline $\begin{array}{l}\text { Failure to } \\
\text { open } \\
\text { valve } B \text {. }\end{array}$ & $\begin{array}{l}\text { Operation } \\
\text { and } \\
\text { maintenance }\end{array}$ & $\begin{array}{l}\text { System fails to take required } \\
\text { action -> } \\
\text { Failure caused by } \\
\text { maintenance -> } \\
\text { Accident would have been } \\
\text { avoided if maintenance } \\
\text { procedure were improved. }\end{array}$ & $\begin{array}{l}\text { The computer control system was designed } \\
\text { to automatically open valve B when flow } \\
\text { was restored to the debutanizer. This } \\
\text { command failed. Subsequent } \\
\text { investigation found of } 39 \text { instrument loops } \\
24 \text { needed attention ranging from minor } \\
\text { mechanical damage to major maintenance } \\
\text { faults. }\end{array}$ \\
\hline
\end{tabular}

Table 2. Abridged IEC 61508 Flowchart Causal Summary for Case Study

\section{Event \& Causal Factor Analysis}

As can be seen, the flowchart analysis in Table 2 is relatively superficial. It provides a causal analysis that might be performed in the initial stages of an investigation. In order to look more closely at detailed design issues, additional questions would be needed in the Flowcharts of Figures 3 and 4 . The resulting diagrams would sacrifice many of the benefits associated with this simple causal analysis technique. The following section, therefore, presents a more sophisticated approach. 


\section{First Stage: Information Elicitation and ECF Modelling}

Figure 5 shows a simplified form of Events and Causal Factors (ECF) diagram. This modeling technique was developed by the US Department of Energy (1992) to provide an overview of events leading to an incident. Rectangles represent events. Ovals represent the conditions that make those events more likely. The diamond shape represents the outcome of the E/E/PES related mishap.

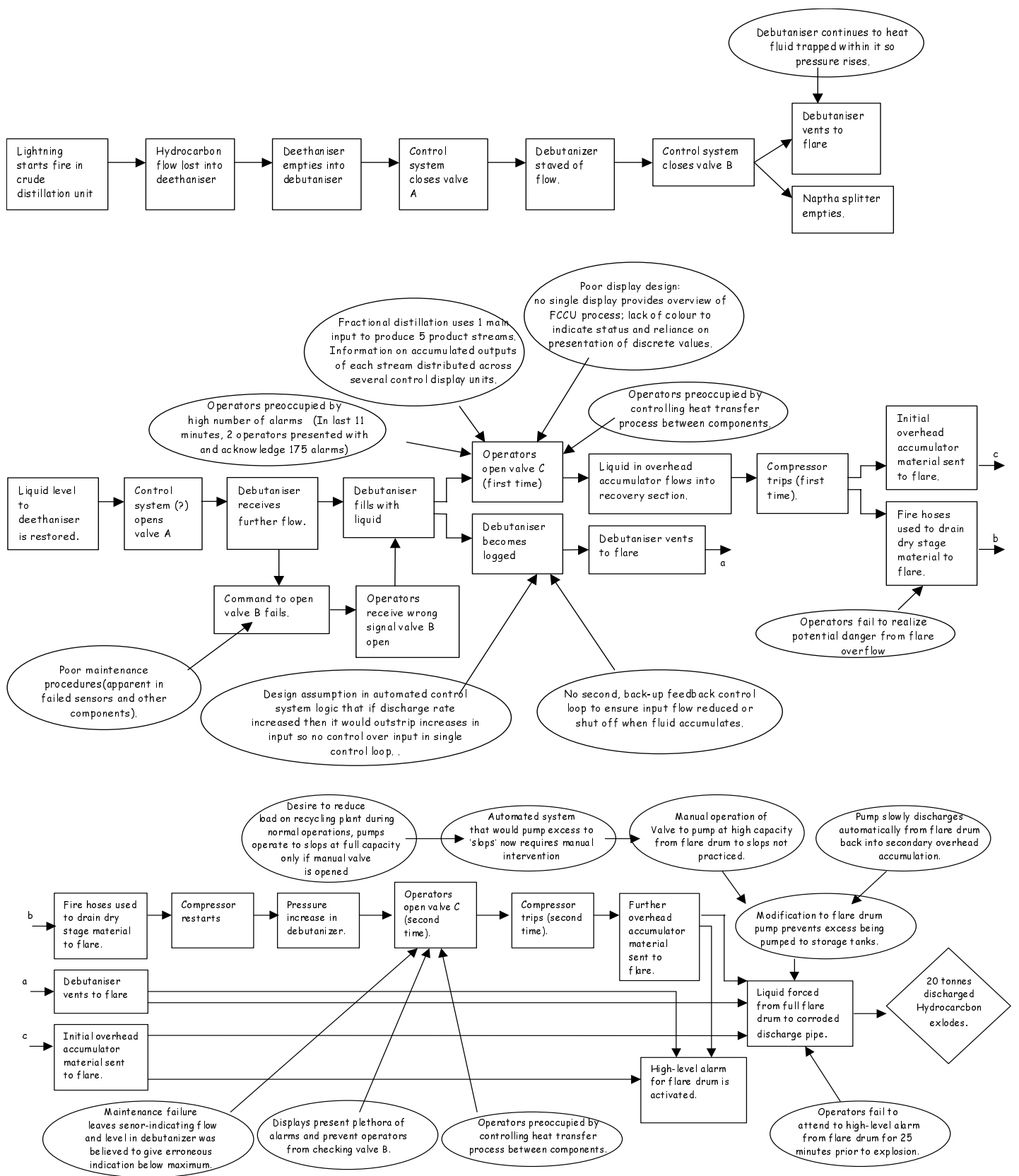

Fig. 5. ECF Diagrams Including Developer/System Integrator Information

This figure is in three parts. The top line represents the chain of events that created the immediate preconditions for the accident. The lightning strike leads to a loss of flow into the debutanizer and an E/E/PES intervened close valve B. The middle line describes a series of intermediate events in which, in 
particular, the E/E/PES fails to open valve B. The flow of materials into the deethanizer and debutanizer creates a build-up, which in turn, leads to materials being passed to the flare.

The middle diagram includes continuation symbols marked $a, b$ and $c$. These feed into the bottom row of the ECF diagram. This illustrates the events and conditions that ultimately lead to the flare drum being filled beyond its capacity so that materials are forced into a corroded discharge pipe and out into the environment. The development of a detailed ECF chart continues until all of the parties involved in an investigation agree that it provides a reasonable representation of the events that contributed to an adverse occurrence or near miss. This decision is influenced by the scope of the investigation and by pragmatics. For instance, we could extend Figure 5 to consider the circumstances that led to 'poor maintenance procedures (apparent in failed sensors and other components)'. This could only be done if incident investigators gain access to the appropriate site documentation or witness statements.

\section{Second Stage: Causal Reasoning}

A further stage of analysis is required in order to distinguish potential causal factors from more contextual information. Starting at the outcome event, investigators must ask whether the incident would have occurred if that event had not taken place. If the incident would still have happened then the event cannot be considered as a casual factor. For example, the incident would arguably not have happened if material had not been forced from the full flare drum into the corroded discharge pipe. This is, therefore, a cause of the incident. Similarly, we can argue that the incident would not have happened if further overhead accumulator material had not been sent to the flare. Conversely, the high-level alarm for the flare had no impact on the course of the incident and so cannot be considered a causal factor. The incident would still have occurred even if the alarm had not sounded.

The causal factors in the ECF diagram are then used to identify potential problems in the development stages and common requirements of IEC 61508, illustrated in Table 1. One means of doing this is to identify the conditions that contributed to each causal event in the ECF chart. These conditions typically capture latent issues, including development and operation decisions that create the context for E/E/Pesrelated mishaps. For instance, the operator's second intervention to open valve $\mathrm{C}$ as a means of reducing pressure in the debutanizer was made more likely by the maintenance failure that prevented them from accurately observing the state of the debutanizer. Poor display design also contributed to their decision, as did their preoccupation with heat transfer within the plant. Heat generated as a by-product of a process was not directly dissipated but was instead used to support other processes in the plant. If either too much or too little heat was generated within the plant then these delicate dependencies that could be disturbed. Table 3 presents some of the results from this analysis. A justification helps others to understand why investigators found violations of common requirements in particular phases of the IEC 61508 lifecycle. Table 3 also included causes that stem from particular stages in the IEC 61508 lifecycle but that are unrelated to any failures in the common requirements.

The causal analysis of our case study illustrates an important point about adverse events involving programmable systems. As can be seen, it is difficult to extract the contribution of computer-related systems from wider failures in the maintenance, operation and safety-management systems. Operators did not intervene to address the automated flare drum alarm because they were busy trying to diagnose the causes of the pressure increase in the debutanizer. They failed to diagnose the problems with the debutanizer because they assumed that the automation had closed valve B. Their task was further exacerbated by their systems' presentation of erroneous sensor readings from the debutanizer. As mentioned, we have exploited the lifecycle and common requirements of IEC 61508 to provide a taxonomy for the causal factors involved in computer-related incidents. This decision was motivated partially by the commercial uptake of this standard and also by the organizational objectives of the specialist unit of the UK Health and Safety Executive who sponsored this work. If another taxonomy were to be used for this purpose then it too would have to support the analysis of incidents in which the failure of programmable devices formed a component of more complex failures in operation, management and the equipment under control. 


\begin{tabular}{|c|c|c|c|c|c|}
\hline $\begin{array}{l}\text { Causal } \\
\text { Event }\end{array}$ & Associated Conditions & $\begin{array}{l}\text { IEC 61508 Lifecycle } \\
\text { Classification }\end{array}$ & Justification & $\begin{array}{l}\text { IEC 61508 Common } \\
\text { Requirements } \\
\text { Violation }\end{array}$ & Justification \\
\hline \multirow{4}{*}{$\begin{array}{l}\text { Liquid } \\
\text { forced from } \\
\text { full flare } \\
\text { drum to } \\
\text { corroded } \\
\text { discharge } \\
\text { pipe. }\end{array}$} & \multirow[t]{3}{*}{$\begin{array}{l}\text { Modification to flare drum } \\
\text { pump prevents excess being } \\
\text { pumped to storage tanks. }\end{array}$} & \multirow[t]{2}{*}{$\begin{array}{l}\text { Modification: } \\
1 \text { impact analysis } \\
\text { incorrect. }\end{array}$} & \multirow{2}{*}{$\begin{array}{l}\text { After modification in normal operation } \\
\text { automated pumps would now reclaim } \\
\text { materials from the flare. Manual } \\
\text { intervention was required to restore high } \\
\text { velocity pumping to slops under } \\
\text { 'emergency' conditions. Operators did } \\
\text { not intervene in this manner and the } \\
\text { impact of this was not considered. }\end{array}$} & $\begin{array}{l}\text { Functional Safety } \\
\text { Assessment: } \\
\text { 2. Modification } \\
\text { assessment LTA. } \\
\end{array}$ & $\begin{array}{l}\text { Assessment of the modification had identified the } \\
\text { need to override low capacity transfer of materials in } \\
\text { flare but had not considered what would happen if } \\
\text { manual intervention did not occur. }\end{array}$ \\
\hline & & & & \multirow{2}{*}{$\begin{array}{l}\text { Verification: } \\
3 \text { LTA verification of } \\
\text { modification }\end{array}$} & \multirow{2}{*}{$\begin{array}{l}\text { There appears not to have been any verification to } \\
\text { determine whether operators could or would } \\
\text { intervene to perform the necessary manual } \\
\text { reconfiguration that was necessary to start high } \\
\text { velocity pump transfer to storage tanks from the } \\
\text { flume tank. }\end{array}$} \\
\hline & & $\begin{array}{l}\text { Modification: } \\
4 \text { LTA verification and } \\
\text { validation }\end{array}$ & $\begin{array}{l}\text { Inadequate testing to see if operators } \\
\text { would intervene once switch was made } \\
\text { away from automated default use of high } \\
\text { velocity pumps to slops. }\end{array}$ & & \\
\hline & $\begin{array}{l}\text { Operators fail to attend to high- } \\
\text { level alarm for flare drum } \\
\text { during } 25 \text { minutes prior to } \\
\text { explosion. }\end{array}$ & $\begin{array}{l}\text { Operation and } \\
\text { maintenance: } \\
9 \text { LTA procedures to } \\
\text { monitor system } \\
\text { performance }\end{array}$ & $\begin{array}{l}\text { Operators were presented with deluge of } \\
\text { automated alarms and lacked } \\
\text { technical/procedural support to } \\
\text { discriminate high priority alarms. }\end{array}$ & \multirow{4}{*}{$\begin{array}{l}\text { Functional Safety } \\
\text { Assessment: } \\
\text { 1. LTA Operations and } \\
\text { Maintenance } \\
\text { assessment. } \\
\text { Safety management: } \\
\text { 2. LTA Safety Audits }\end{array}$} & \multirow{4}{*}{$\begin{array}{l}\text { The incident was caused by a number of problems in } \\
\text { the way in which the system was both maintained } \\
\text { and operated. Maintenance failures meant that } \\
\text { automated systems and operators could not rely on } \\
\text { some sensor readings. The tight integration of heat } \\
\text { transfer operations together with poor alarm } \\
\text { handling created immense burdens for system } \\
\text { operators under abnormal situations and these } \\
\text { demands appear not the have been assessed in a } \\
\text { systematic manner. }\end{array}$} \\
\hline \multirow[t]{3}{*}{$\begin{array}{l}\text { Operators } \\
\text { open valve } \\
\text { C }\end{array}$} & $\begin{array}{l}\text { Maintenance failure leaves } \\
\text { sensor indicating that the flow } \\
\text { and level in the debutanizer } \\
\text { was believed to give erroneous } \\
\text { indication below maximum. }\end{array}$ & $\begin{array}{l}\text { Operation and } \\
\text { maintenance: } \\
\text { 2: maintenance } \\
\text { procedures need } \\
\text { improvement } \\
\end{array}$ & $\begin{array}{l}\text { The programmable systems and operator } \\
\text { alarms depended on accurate sensor } \\
\text { information. Inadequate maintenance } \\
\text { created systemic vulnerabilities that were } \\
\text { likely to lead to mishaps. }\end{array}$ & & \\
\hline & $\begin{array}{l}\text { Display presents plethora of } \\
\text { alarms that prevent operators } \\
\text { from checking status of valve } \\
\text { B. }\end{array}$ & $\begin{array}{l}\text { Allocation: } \\
\text { 4. Installation design }\end{array}$ & $\begin{array}{l}\text { Operators had to acknowledge almost } \\
400 \text { alarms in the last } 12 \text { minutes of the } \\
\text { mishap. This took away from time to } \\
\text { diagnose the problem and plan their } \\
\text { intervention. }\end{array}$ & & \\
\hline & $\begin{array}{l}\text { Operator preoccupied by } \\
\text { controlling heat transfer } \\
\text { process between components }\end{array}$ & $\begin{array}{l}\text { Overall safety } \\
\text { requirements: } 4 . \\
\text { Installation design. }\end{array}$ & $\begin{array}{l}\text { Heat generated as a by-product of one } \\
\text { sub-process was used elsewhere in the } \\
\text { system rather than dissipated by cooling } \\
\text { systems. This created delicate } \\
\text { dependencies that would be disturbed } \\
\text { and impose additional burdens on } \\
\text { operators during emergency situations. }\end{array}$ & & \\
\hline
\end{tabular}

Table 3. IEC 61508 Causal Summary Chart for Case Study Incident 
Chris Johnson,

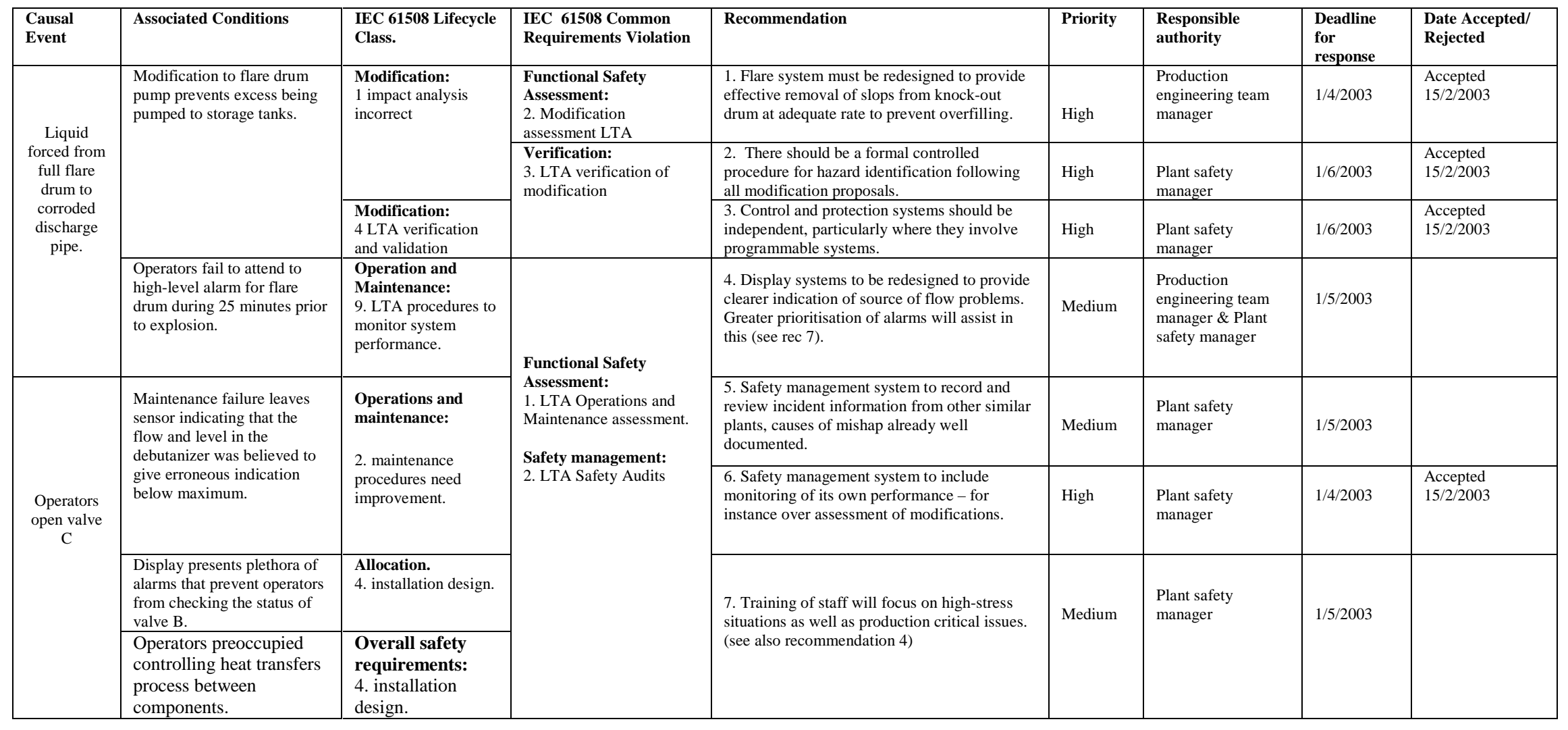

Table 4. Recommendation Summary Form (LTA - Less Than Adequate) 


\section{Generating Recommendations}

The final activity in our simple and complex causal analysis techniques is to produce recommendations that help to avoid any recurrence of an incident or near miss. The generation of recommendations uses the outcome of previous stages to identify potential recommendations. These recommendations are clearly domain and incident dependent. It is important, however, that investigators document the actions that are intended to avoid any recurrence of an incident involving programmable systems. Each recommendation should be associated with a priority assessment, with an individual or organization responsible for implementing it and with a potential timescale for intervention. Typically, a safety manager will then respond with a written report stating whether each recommendation has been accepted or rejected (Johnson, 2003).

It is important when drafting a recommendation that investigators consider whether similar interventions have been advocated in the past. Electronic information systems can be used to assist in this task. The key point, however, is that ineffective recommendations should not continue to be issued in the face of recurrent incidents. Similarly, it is important to identify situations in which recommendations are consistently rejected or inadequately implemented. Any accepted recommendations must be disseminated to those who are responsible for acting upon them. Safety managers must also assume responsibility for checking that any necessary changes are implemented according to the agreed timescale. System documentation must be updated to reflect any subsequent modifications. Table 4 provides an example of a form that can be used to record recommendations from incidents involving programmable systems. As can be seen, different deadlines may be associated with actions that have different priority levels. This does not imply that high priority items will have an immediate deadline. Additional time is often necessary to ensure that subsequent interventions do not introduce further flaws in the design, operation and maintenance of safety-critical systems.

A key concern behind the design of Tables 3 and 4 is that investigators should be accountable for their recommendations. By this we mean that co-workers, safety managers and regulators should be able to trace back particular recommendations through the previous stages of any causal analysis so that it is possible to identify the reasons why particular interventions are proposed in the aftermath of an adverse event. For example, recommendation 4 proposes a redesign of the control system displays. This is based on the observation that operations and maintenance assessments had been less than adequate prior to the incident. In particular, these assessments had failed to predict the impact that multiple alarms had upon their ability to correctly diagnose the status of valve B. If they had not been forced their observation of multiple low priority warnings then they might have been better able to recognize that their control system had failed to complete their command to open the flow from the debutanizer.

\section{Conclusions}

A range of techniques has been developed to support the analysis and investigation of adverse events and near miss incidents. Very few of these techniques have been specifically designed to support the investigation of incidents involving programmable systems. This report, therefore, introduces two investigation methods for this class of adverse events. The first builds on a relatively simple flowchart. Investigators can identify and categorize the causes of a mishap by answering a series of questions. The responses that they provide guide the causal analysis to underlying problems in the design, development or operation of E/E/PES hardware and software.

The second, more complex, approach introduces several additional stages of analysis. It is appropriate for more complex incidents where the questions that guide a simpler form of analysis may not be 
directly applicable. These additional stages also provide intermediate documentation that is necessary when investigators must justify their conclusions to other investigators, safety managers and courts of law. In particular, this second approach relies upon a timeline reconstruction of an adverse event using a technique known as Events and Causal Factors (ECF) charting. This produces a graphical sketch of the events leading to an incident. This can then be used to distinguish contextual information from causal factors. In our proposed method, these causal factors are then analyzed to identify potential failures in the lifecycle of programmable systems using a checklist approach.

Both of our investigation techniques have been tailored to provide information that guides the future development and operation of safety-critical systems. In particular, the flowchart and checklist help investigators to map from the causes of hardware and software related incidents to the clauses of the IEC 61508 standard. IEC 61508 provides guidance on the activities that should be conducted during the concept development, overall scoping, hazard and risk assessment, overall safety requirements analysis, integration, commissioning and verification, realization, validation, operation and maintenance, and modification of safety critical computer systems. In addition there are a range of requirements that are common to all lifecycle phases. These include the need to ensure the competency of those involved in the operation, maintenance and modification of the system. They also include requirements relating to the 'safety culture' of the organizations involved in the development and operation of programmable systems. Our use of this standard is justified because it provides a means of feeding the insights derived from any incident investigation back into the future maintenance and development of hardware and software within safety-critical applications.

There are a number of directions for future work. In particular, previous sections have argued that our taxonomy provides insufficient support for the analysis of human factors issues, including display design and task allocation. This concern could be addressed by introducing an additional human factors component under the common requirements of table 1. More detailed categories could include 'LTA identification of human actions or errors leading to hazards', 'human failure to respond effectively to alarms or take other necessary operational action due to deficient design', 'human failure in testing and maintenance due to inadequate procedures or design'. It would also be possible to introduce additional questions into the flowchart of Figures 3 and 4. This would be justified even though there is a danger that the gradual introduction of additional elements will complicate the simplified analysis technique. The key point here is that our techniques should be refined to reflect particular operational needs as experience in incident investigation reveals potential omissions in the taxonomy and flow chart.

Our techniques are likely to identify incidents that cannot easily be attributed to lifecycle phases or common requirements in IEC 61508. The link between constructive design standards and analytical investigation techniques can, therefore, yield insights into the limitations of these standards. An implicit motivation in our work is to provide the feedback mechanisms that are necessary to improve the application of standards, such as IEC 61508 and DO-178B.

\section{Acknowledgements}

Thanks are due to Bill Black (Black Safe Consulting), Mark Bowell (UK HSE) and Peter Bishop (Adelard) for providing comments on the initial draft of this document.

\section{References}

P.G. Bishop, R.E Bloomfield, L.O. Emmet, C.W. Johnson, W. Black, V. Hamilton and F. Koornneef (2003 in press), Learning from Incidents - Outline Scheme for E/E/PE Safety-Related Systems, HSE Contract Research Report. 
Department of Energy, DOE Guideline Root Cause Analysis Guidance Document, Office of Nuclear Energy and Office of Nuclear Safety Policy and Standards, U.S. Department of Energy, Washington DC, USA, DOE-NESTD-1004-92, http://tis.eh.doe.gov/techstds/standard/nst1004/nst1004.pdf, 1992.

HSE (1997), The Explosion and Fires at the Texaco Refinery, Milford Haven, 24 ${ }^{\text {th }}$ July 1994 . HSE Books, Norwich, UK.

International Electrotechnical Commission (2003), IEC 61508 Functional Safety of Programmable Electronic Safety-Related Systems. Available via http://www.iec.ch/functionalsafety

C.W. Johnson (2003 in press), A Handbook for the Reporting of Incidents and Accidents, Springer Verlag, London, UK.

C.W. Johnson, G. Le Galo and M. Blaize, (2000), Guidelines for the Development of Occurrence Reporting Systems in European Air Traffic Control, European Organisation for Air Traffic Control (EUROCONTROL), Brussels, Belgium.

P. Ladkin and K. Loer (1998), Why-Because Analysis: Formal Reasoning About Incidents, Bielefeld, Germany, Document RVS-Bk-98-01, Technischen Fakultat der Universitat Bielefeld, Germany.

N. Leveson, (2002), A Systems Model of Accidents. In J.H. Wiggins and S. Thomason (eds) Proceedings of the $20^{\text {th }}$ International System Safety Conference, 476-486, International Systems Safety Society, Unionville, USA. 